\title{
The MELAS phenotype may not only be determined by heteroplasmy of causative mtDNA variants
}

\author{
Josef Finsterer $(\mathbb{D}$ \\ Klinik Landstrasse, Messerli Institute, Vienna, Austria
}

Correspondence

should be addressed

to J Finsterer

Email

fifigs1@yahoo.de

I read with interest, the article by Chae et al. about a study of 32 paediatric patients with MELAS of whom 25 underwent next-generation sequencing (NGS) (1). It was concluded that heteroplasmy rates correlated inversely with age at onset and age at diagnosis (1). This raises the following comments and concerns.

The main shortcoming of the study is that heteroplasmy rates were determined in blood lymphocytes, which are usually not severely affected in MELAS. Thus, heteroplasmy rates in blood lymphocytes may not represent heteroplasmy rates in other more severely affected tissues. This is why the results provided remain questionable. Heteroplasmy rates should also be determined in tissue such as buccal mucosa cells, hair follicles, urine epithelial cells, skin fibroblasts, or the skeletal muscle.

A second shortcoming is that the phenotypic expression of the m.3243A $>\mathrm{G}$ variant may not only depend on heteroplasmy rates but also on the mtDNA copy number, on the haplotype, and on polymorphisms. Thus, the authors should provide at least mtDNA copy numbers to assess if the severity of the phenotype depended on the amount of available mtDNA or not.

Furthermore, heteroplasmy rates may depend on the disease stage, which is why it is important to know if heteroplasmy rates were determined only once or repeatedly during follow-up. Heteroplasmy rates may increase over time even in less severely affected tissues.

Also missing is the current medication the 25 included patients received. As a number of drugs (e.g. steroids, antibiotics) may influence the phenotype of mitochondrial disorders (MIDs), it is crucial to know the drugs the included patients were regularly taking.

According to Table 1, the family history was positive in seven patients (1). However, if this means that the mothers of these seven patients had MELAS or that they had diabetes without MELAS is not reported.

MELAS may not only manifest in the CNS, eyes, ears, endocrine organs, heart, intestines, or kidneys, but also in the vestibular organs (2), as sudden unexpected death (3), and in peripheral nerves (4). How many of the 25 patients included had vestibular involvement or neuropathy is not reported as well as in how many of them neuropathy was attributed to diabetes, with reference to the underlying mutation.

Overall, the study has a number of shortcomings which need to be addressed before the conclusions drawn can be justified. Heteroplasmy rates in blood lymphocytes may not be related to phenotypic severity and the phenotype may not only be determined by heteroplasmy rates.

\section{Declaration of interest}

The author declares that there is no conflict of interest that could be perceived as prejudicing the impartiality of this letter.

\section{Funding}

This work did not receive any specific grant from any funding agency in the public, commercial or not-for-profit sector.

\section{Informed consent}

The study was approved by the institutional review board. (c) 2021 European Society of Endocrinology Printed in Great Britain
Published by Bioscientifica Ltd. 


\section{Author contribution statement}

J F was involved in the design, literature search, discussion, first draft and critical comments.

\section{References}

1 Chae HW, Na JH, Kim HS \& Lee YM. Mitochondrial diabetes and mitochondrial DNA mutation load in MELAS syndrome. European Journal of Endocrinology 2020183 505-512. (https://doi.org/10.1530/ EJE-20-0189)
2 Iwasaki S, Egami N, Fujimoto C, Chihara Y, Ushio M, Kashio A \& Yamasoba T. The mitochondrial A3243G mutation involves the peripheral vestibule as well as the cochlea. Laryngoscope $2011 \mathbf{1 2 1}$ 1821-1824. (https://doi.org/10.1002/lary.21879)

3 Reed EM, Swanson AA, Roden AC \& Lin PT. Sudden unexpected death in MELAS syndrome due to diabetic ketoacidosis. American Journal of Forensic Medicine and Pathology 202041 331-332. (https:// doi.org/10.1097/PAF.0000000000000563)

4 Chu CC, Huang CC, Fang W, Chu NS, Pang CY \& Wei YH. Peripheral neuropathy in mitochondrial encephalomyopathies. European Neurology 199737 110-115. (https://doi. org/10.1159/000117420)

Received 29 October 2020

Revised version received 26 November 2020

Accepted 14 December 2020 